\title{
Morphological and Chemical Evolution of Silicon Nanocomposite during Cycling
}

\author{
Mahsa Sina ${ }^{1}$, Judith Alvarado ${ }^{2}$, Hitoshi Shobukawa ${ }^{1}$, Ying Shirley Meng ${ }^{1,2}$ \\ ${ }^{1}$ Department of NanoEngineering, University of California San Diego, La Jolla, California, United \\ States \\ ${ }^{2}$ Materials Science \& Engineering Program, University of California San Diego, La Jolla, California, \\ United States
}

High-capacity materials are required to further the development of Li-ion batteries. As a result, silicon (Si) has been investigated as a promising anode in Li-ion batteries because of its high theoretical capacity $(3579 \mathrm{mAh} / \mathrm{g})$, which is 10 times higher than of the commercial graphite anodes $(372 \mathrm{mAh} / \mathrm{g})$. However, Si electrodes undergo sever mechanical degradation due to the volume exaptation $(300 \%)$ upon lithiation and suffers from an unstable solid electrolyte interphase (SEI) layer. The SEI layer occurs at the electrode/electrolyte interface as a result of the electrolyte decomposition. In this work crystalline $\mathrm{Si}$ (60 $\mathrm{nm}$ particle size) was used to prevent large strain that occurs from the volume expansion. In order to improve the cycling performance of $\mathrm{Si}$ composite electrode, fluoroethylene carbonate (FEC) electrolyte additive was added to the electrolyte [1-4]. For the first time, various TEM/STEM EELS techniques demonstrated the effects of FEC on the morphological and chemical evolution of the SEI on Si composite electrode during electrochemical cycling.

ADF-STEM images of the lithiathed electrodes cycled in EC/DEC and EC/DEC/FEC are shown in Figure 1. The surface of the lithiated electrode cycled in EC/DEC (Figure 1(a)) is unevenly covered with a porous SEI layer. Conversely, adding $10 \mathrm{wt} \% \mathrm{FEC}$ to the traditional (EC/DEC) electrolyte forms a uniform dense SEI while maintaining particle integrity. (Figure 1(c)). The corresponding EELS spectra (Figure 1(e)) from the lithiated electrodes with and without FEC further confirm the presence of a SEI layer. The EELS spectra results demonstrate that the SEI layer (darker area) does not contain Si. Additionally, the SEI mainly contains $\mathrm{Li}_{2} \mathrm{CO}_{3}$ for the electrode cycled in EC/DEC, however, it mostly consisting of $\mathrm{LiF}$ for the electrode cycled in EC/DEC/FEC. Throughout electrochemical cycling $(1,5$ and 100 cycles) the SEI layer on the electrode cycled with EC/DEC thickens unevenly (Figure 1(b)) were the electrode cycled in EC/DEC/FEC formed uniform dense SEI up to 5 cycles. Surprisingly, the electrode cycled with FEC formed a thick inhomogeneous SEI after 100 cycles.

Figure 2 shows the comparison of the EELS spectra from the cycled Si electrode in EC/DEC and $\mathrm{EC} / \mathrm{DEC} / \mathrm{FEC}$ after various cycle numbers. The Si-L varies significantly between bulk and surface of the $\mathrm{Si}$ cycled in EC/DEC, which is attributed to the presence amorphous $\mathrm{Li}_{\mathrm{x}} \mathrm{Si}$ alloy and $\mathrm{Li}_{\mathrm{x}} \mathrm{SiO}_{\mathrm{y}}$ in the bulk and at the edge of the electrode (Figure 2(a)). After one cycle, only amorphous $\mathrm{Li}_{\mathrm{x}} \mathrm{Si}$ was detected at the surface and bulk of the $\mathrm{Si}$ cycled in $\mathrm{EC} / \mathrm{DEC} / \mathrm{FEC}$ and the $\mathrm{Li}_{\mathrm{x}} \mathrm{SiO}_{\mathrm{y}}$ was not detected at the edge (Figure 2(b)). However, the $\mathrm{Li}_{\mathrm{x}} \mathrm{SiO}_{\mathrm{y}}$ was observed after 5 cycles and increased at the interface of the electrode cycled in EC/DEC/FEC with cycle number. Interestingly, the presence of FEC suppresses the formation of $\mathrm{Li}_{x} \mathrm{SiO}_{\mathrm{y}}$ layer and also it leads to the formation of uniform and homogenous SEI layer with high $\mathrm{LiF}$ content as a result of the initial FEC decomposition. Pushing the boundaries of the STEM/EELS led to further understanding of the effects that FEC has on the SEI formation by directly visualizing and characterizing Si composite electrodes [5]. 


\section{References:}

[1] Philippe B., et. al., G. G. (2013), Chemistry of Materials, 25: 394-404.

[2] Xu C., F., et al. (2015), Chemistry of Materials, 27: 2591-2599.

[3] McDowell M. T., et al. (2012), Advanced Materials 24: 6034-6041.

[4] Schroder K., et. al. (2015), Chemistry of Materials, 27: 5531-5542.

[5] This research is supported by the Office of Vehicle Technologies, U.S. Department of Energy under Contract No. DE-AC02-05CH11231, Subcontract No. 7073923 under the Advanced Battery Materials Research (BMR) Program.
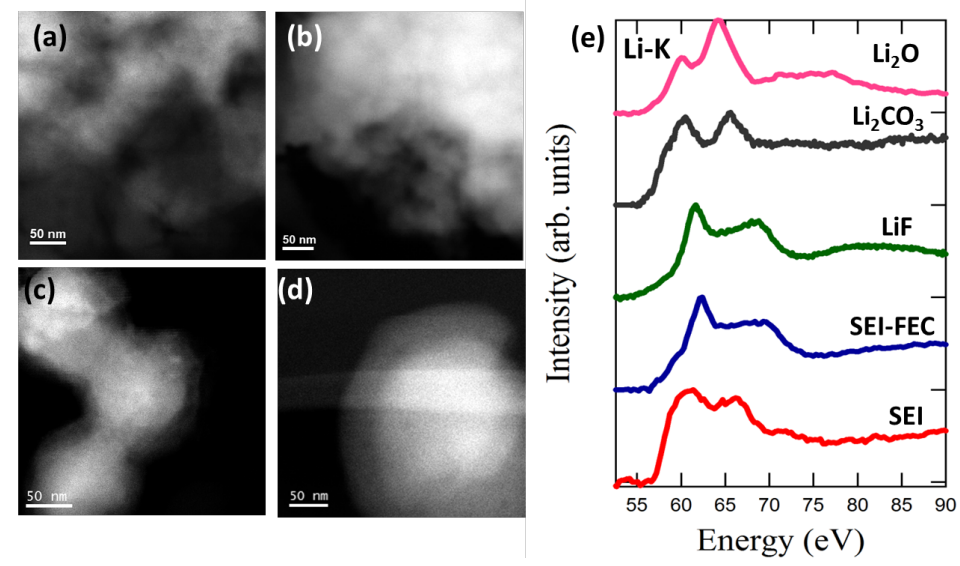

Figure 1. ADF STEM images of the lithiated electrodes cycled with EC/DEC at the (a) first cycle and (b) five cycles. Lithiated Si composite electrodes cycled with EC/DEC/FEC at (c) first cycle and, (d) five cycles. (e) The corresponding Li-K edge EELS spectra taken from SEI layer of the lithiated Si with and without FEC comparing the possible standard phases ( $\mathrm{LiF}, \mathrm{Li}_{2} \mathrm{CO}_{3}, \mathrm{Li}_{2} \mathrm{O}$ ).
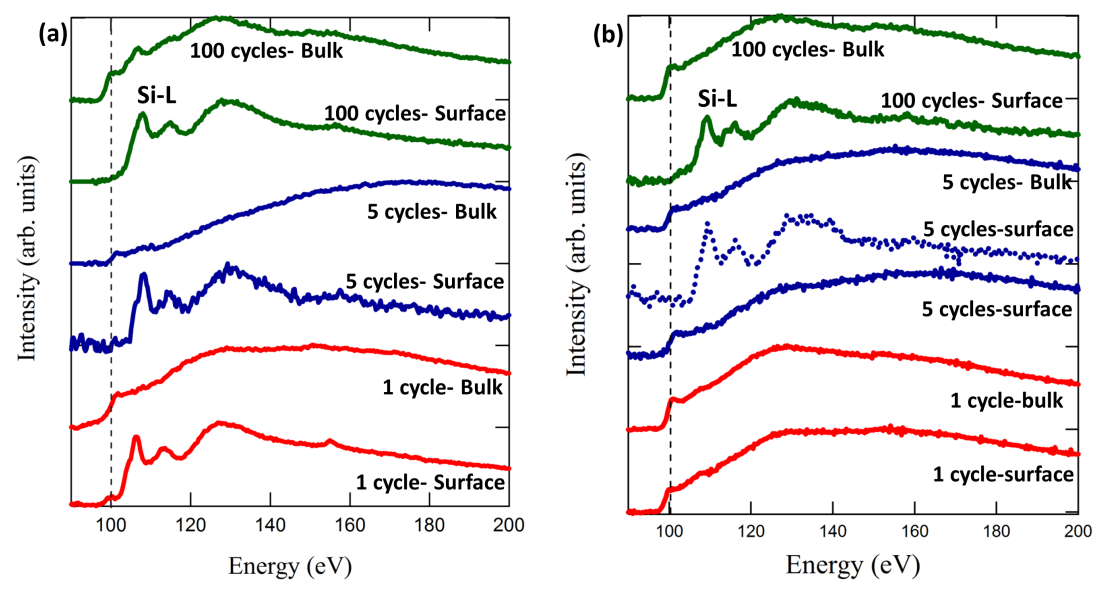

Figure 2. Si-L EELS spectra of the Si electrodes cycled in (a) EC/DEC and (b) EC/DEC/FEC at various cycle numbers. 